\title{
Plasmonic Filter Arrays for Infrared Spectral Reconstruction
}

\author{
Benjamin Craig, Vivek Raj Shrestha, Jiajun Meng, and Kenneth B. Crozier \\ School of Physics and Department of Electrical and Electronic Engineering, University of Melbourne, Victoria 3030, Australia \\ bcraig@student.unimelb.edu.au, kenneth.crozier@unimelb.edu.au
}

\begin{abstract}
We experimentally demonstrate an array of plasmonic infrared transmission filters. We show, by simulations that include noise, reconstruction of the spectra of infared light sources from measurements of the energy transmitted through each filter.

OCIS codes: (250.5403) Plasmonics; (300.6340) Spectroscopy; (080.1753) Computation methods; (040.3060) Infrared
\end{abstract}

\section{Introduction}

Many applications of infrared (IR) spectroscopy would benefit from spectrometers that are smaller, lighter and less expensive than the current workhorse tool, the Fourier transform infrared (FTIR) spectrometer. There has progress recently on the realization of spectrometers comprising filter arrays combined with photodetector arrays. It has been shown that the spectrum of the (unknown) incident light source can be numerically reconstructed from the photodetector signals, provided that the filter functions are known. Demonstrations have been made at visible wavelengths using filters comprising quantum dots or thin film etalons [1, 2]. Here we demonstrate the creation of a two dimensional (2D) array of plasmonic filters that span the $4-14 \mu \mathrm{m}$ range. Integration of our device with a 2D IR detector array (eg. microbolometers) would enable realization of a compact, lightweight and low cost spectrometer. We demonstrate, by simulations, the spectral reconstruction of two infrared light sources.

\section{Filter Design and Fabrication}

Each filter is a 2D array of Au square patches (30 nm thick) formed on an undoped silicon substrate (Fig. 1a), with Pd for adhesion. Each square Au patch has a side length $(P / 2)$ that is half the array period $(P) .151$ filters are fabricated, with $P$ varying from 1 to $4 \mu \mathrm{m}$, in steps of $20 \mathrm{~nm}$. Each filter has an overall extent of $100 \mu \mathrm{m} \times$ $100 \mu \mathrm{m}$. The filters are fabricated by electron beam lithography, evaporation and lift-off. Optical and scanning electron microscope images of fabricated devices are shown as Fig. 1b and 1c.
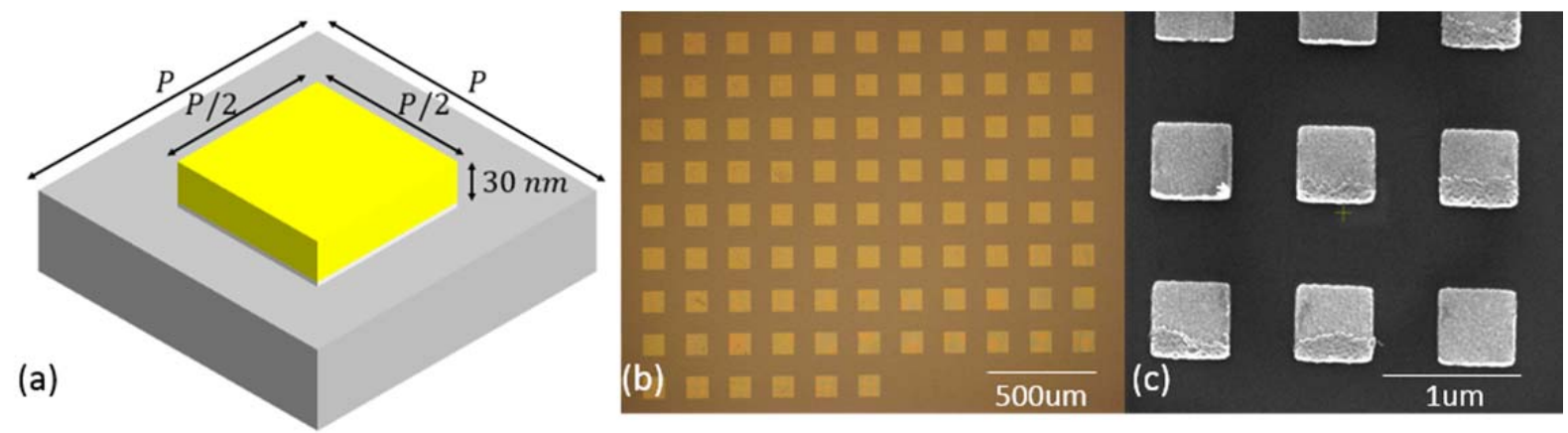

Fig. 1. (a) Unit cell of filter. (b) Optical microscope image of device (94 filters shown). (c) SEM image of part of filter with $P=1 \mu m$.

\section{Results}

Transmission measurements are performed using an FTIR microscope, with the results for the $P=1 \mu m$ device shown as Fig. 2a along with the finite difference time domain (FDTD) simulation. Both measured and simulated spectra are absolute transmission. For the measured spectrum, this is obtained by dividing the transmission measured through the sample by that measured through air. For the simulated spectrum, this is obtained by multiplying the simulated power transmission spectrum of an unpatterned air-to-silicon interface by the simulated transmission spectrum of a silicon-to-air interface on which the 2D gold square array is formed. It can be seen that experiment and simulation show a spectral dip, and are good agreement. No fitting parameters are used in the simulations. The minor differences between simulations and experiments (Fig. 2a) might be due to the fact that the simulations assume purely normal incidence and due to differences between fabricated and simulated devices regarding roughness (e.g. see Fig. 1c) and the optical properties of the materials. Transmission spectra of selected and all devices are shown as Fig. 2b and 2c, respectively. We also note that it can be seen that the transmission 
spectra of the filters with periods from $1.8-2.2 \mu \mathrm{m}$ exhibits some features in the $10-11 \mu \mathrm{m}$ spectral range. It is believed that these originate from detector instability. This will be subject of future investigation.
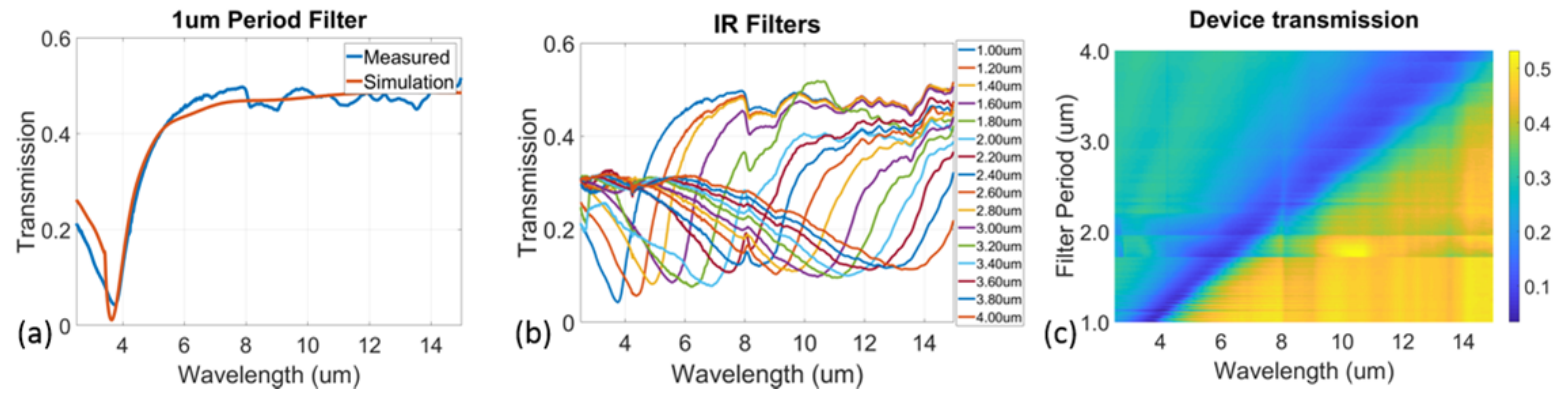

Fig. 2. (a) Measured and simulated transmission spectra of 1um period filter. (b) Measured transmission of 16 of the 151 filters, whose periods $(P)$ are as indicated. (c) 2D plot of measured transmission for all filter, showing clear linear shifting and broadening of dips as period increases.

We demonstrate, through simulations that include simulated noise, the spectral reconstruction of two light sources using the recursive least square (RLS) method [3], which in general takes input signals and recursively finds the coefficients that minimize a weighted linear least cost function relating to the inputs. This is performed with the goal of demonstrating that our device could be used with an array of 151 energy detectors to determine the spectra of unknown light sources. Our demonstration is performed as follows. Our MATLAB code first randomly generates an arbitrary source spectrum (blue curve Fig. 3a). Using the measured transmission spectra of our device (Fig. 2c), the program predicts the energy that would be transmitted through each of 151 filters. The program then adds noise and the resultant energies are input to our reconstruction algorithm along with the transmission spectra (Fig. 2c). The spectra reconstructed (with different noise levels) in this way can be seen to be in good agreement with the original source spectrum (Fig. 3a). We note that low pass filtering is applied to the reconstructed spectra to mitigate high frequency noise. We repeat this process, this time with the original source spectrum comprising the spectrum of the globar source in our FTIR spectrometer measured by the internal deuterated tri glycine sulfate (DTGS) detector. It can seen that the reconstructed spectra are again in good agreement with the original source (Fig. 3b).
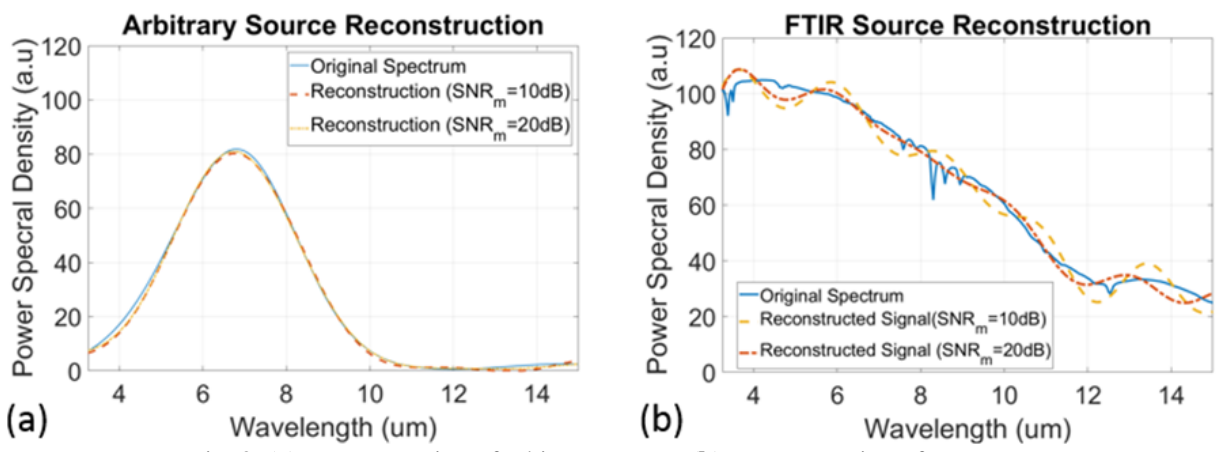

Fig. 3. (a) Reconstruction of arbitrary source. (b) Reconstruction of FTIR source

In summary, we experimentally demonstrate long- to mid-wave IR filters based on arrays of Au square patches on silicon substrates. While filters over wavelengths from $4-14 \mu \mathrm{m}$ are demonstrated, we note that there is no reason that this spectral range could not be extended. We report simulations that demonstrate that our device could be used with a detector array for the spectral reconstruction of various sources. Future work will involve experimentally reconstructing the FTIR globar source, testing alternate plasmonic filter designs and creating a more sophisticated reconstruction program.

\section{References}

[1] E. Huang, Q. Ma and Z. Liu, "Spectrum Engineering for Reconstructive Spectrometry," in CLEO conference, San Jose, CA, 2016 , pp. 1-2.

[2] J. Bao and M. G. Bawendi, "A colloidal quantum dot spectrometer," Nature, vol. 523, 67-70 (2015).

[3] M. H. Hayes, "9.4: Recursive Least Squares," in Statistical Digital Signal Processing and Modeling, Wiley, 1996, p. 541.

Performed in part at Melbourne Centre for Nanofabrication in the Victorian Node of the Australian National Fabrication Facility (ANFF). 